\title{
Deficiência de vitamina B12 em pacientes de uma enfermaria de clínica médica em Fortaleza/CE
}

\section{Vitamin B12 deficiency in patients of a medical clinic nursing in Fortaleza/CE}

Jéssica Maria Moura Cassimiro ${ }^{1}$. Claudio Abreu Barreto Junior². Gabriella Pequeno Costa Gomes de Aguiar ${ }^{3}$. Otilio José Nicolau de Oliveira 4 .

1 Graduanda do sexto ano de Medicina da Universidade Estadual do Ceará, Fortaleza, Ceará, Brasil. 2 Graduando do quinto ano de Medicina da Universidade Estadual do Ceará, Fortaleza, Ceará, Brasil. 3 Residente em Clínica Médica do Hospital Geral de Fortaleza, Ceará, Brasil. 4 Preceptor de Clínica Médica do Hospital Geral de Fortaleza, Ceará, Brasil

\section{RESUMO}

Foi realizado um estudo transversal que contou com 116 participantes que estiveram internados na enfermaria de Clínica Médica do Hospital Geral de Fortaleza (HGF) nos seis primeiros meses do ano de 2015. O objetivo foi descrever a prevalência da deficiência de vitamina B12 e descrever as características clínicas em pacientes internados nessa unidade. A prevalência de deficiência de cobalamina foi de $18 \%$. Encontramos que $40 \%$ dos pacientes apresentaram afecção neurológica não explicada por causas vasculares, $20 \%$ anemia macrocítica, $20 \%$ alcoolismo, $20 \%$ uso de inibidor de bomba de prótons, $10 \%$ uso de metformina, $10 \%$ dieta estritamente vegetariana, $10 \%$ diagnóstico de infecção por HIV, 10\% diagnóstico prévio de gastrite atrófica e $10 \%$ diagnóstico prévio de hipotireoidismo. Em nenhum caso supracitado encontrou-se significância estatística $(\mathrm{p}<0,05)$.

Palavras-chave: Deficiência de vitamina B12. Região tropical. Cianocobalamina.

\section{ABSTRACT}

A cross-sectional study included 116 participants who were hospitalized in the Internal Medicine ward of the General Hospital of Fortaleza (HGF) in the first six months of 2015. The objective was to describe the prevalence of vitamin B12 deficiency and describe the features clinics in patients admitted in this unit. The prevalence of cobalamin deficiency was $18 \%$. We found that $40 \%$ of patients had neurological condition not explained by vascular causes, $20 \%$ macrocytic anemia, $20 \%$ alcohol, $20 \%$ use of proton pump inhibitor, $10 \%$ use of metformin, $10 \%$ strictly vegetarian diet, $10 \%$ diagnostic $\mathrm{HIV}$ infection, $10 \%$ previous diagnosis of atrophic gastritis and $10 \%$ prior diagnosis of hypothyroidism. In any case above was found statistically significant $(\mathrm{p}<0.05)$.

Keywords: Vitamin B12 deficiency. Tropical region. Cyanocobalamin.

Autor correspondente: Jéssica Maria Moura Cassimiro, Rua João Cordeiro, 944, apartamento 803, Meireles, Fortaleza, Ceará.Telefone: +5585 3055-6803/+55 88 99604-6671. E-mail: jessicammcassimiro@gmail.com

Conflito de interesses: Não há qualquer conflito de interesses por parte de qualquer um dos autores.

Recebido em: 07 Dez 2015; Revisado em: 07 Mar 2016; Aceito em: 23 Mai 2016. 


\section{INTRODUÇÃO}

A vitamina B12 (cianocobalamina) faz parte de uma família de compostos, que são genericamente denominados cobalaminas. É uma vitamina hidrossolúvel, sintetizada exclusivamente por micro-organismos e estocada primariamente no fígado. Sua fonte natural de obtenção é a dieta rica em alimentos de origem animal..$^{1-3}$

Em países em desenvolvimento, como Índia, México e Guatemala, verificou-se alta prevalência de deficiência de cobalamina em gestantes e mulheres em amamentação. Além disso, estudos no México, Venezuela e Quênia mostraram baixa concentração sérica de cobalamina em 33$52 \%$ das crianças. ${ }^{4,5} \mathrm{Um}$ estudo em que participaram 288 famílias mexicanas realizou investigação longitudinal dos efeitos da má nutrição e demonstrou alta prevalência de deficiência de vitamina B12 em todas as idades e ambos os gêneros. ${ }^{4}$

Sabe-se que a incidência dessa deficiência aumenta de forma significativa com a idade. ${ }^{6,7} \mathrm{Em}$ países industrializados, calcula-se que aproximadamente $20 \%$ da população idosa apresenta deficiência de vitamina $\mathrm{B} 12 .{ }^{8}$ É possível que a prevalência dessa carência vitamínica seja subestimada, pois há importante variação de sensibilidade e especificidade entre as metodologias laboratoriais..$^{9,10}$

A dieta ocidental usual contém em média 5 a $7 \mathrm{mcg}$ de cianocobalamina por dia. Essa quantidade é, em geral, suficiente para manter o equilíbrio normal da cobalamina sérica. A ingesta diária recomendada deste micronutriente varia de acordo com o grupo populacional estudado. Em adultos, recomenda-se uma ingesta diária de no mínimo 2 mcg por dia. Por outro lado, esta quantidade deve aumentar em gestantes e lactantes para 2,6 mcg por dia. Em populações mais jovens, o número varia de $0,7 \mathrm{mcg}$ em crianças a $2 \mathrm{mcg}$ em adolescentes. ${ }^{11,12}$

Diante desses dados e da escassez de estudos científicos que caracterizem o perfil dos portadores da deficiência de vitamina B12 no nosso meio, esse trabalho objetivou analisar sua prevalência e descrever as características clínicas em uma enfermaria de Clínica Médica em Fortaleza/CE.

\section{METODOLOGIA}

O estudo possui caráter transversal e prospectivo tendo sido realizado entre os meses de janeiro a junho de 2015. Foram incluídos vinte participantes por mês da amostra seguindo os seguintes critérios de inclusão: pacientes internados na enfermaria de Clínica Médica do Hospital Geral de Fortaleza; pacientes escolhidos randomicamente no período avaliado; pacientes que assinaram o termo de consentimento livre e esclarecido. A randomização dos pacientes foi realizada por meio do programa de tabulação e análise de dados SPSS 22 IBM $^{\circledR}$.

A pesquisa foi avaliada e aprovada pelo Comitê de Ética em Pesquisa do HGF e está de acordo com os critérios estabelecidos pela Declaração de Helsinki e com as suas modificações.

O rastreio inicial foi realizado com dosagem dos níveis séricos de vitamina B12 e homocisteína. A partir de então, apenas os pacientes com dosagem de vitamina B12<200 pg/mL e/ou homocisteína $>15$ umol/L seguiram em investigação.

Por meio da anamnese e revisão do prontuário foram pesquisados os seguintes fatores de risco: idade, alcoolismo, dieta, uso de metformina na dose mínima de $500 \mathrm{mg}$ por dia pelo tempo mínimo de 16 semanas, uso de inibidores de bomba de prótons na dose mínima de $20 \mathrm{mg}$ por dia pelo tempo mínimo de 08 semanas, cirurgia gastrointestinal anterior, diagnóstico prévio de HIV, doença celíaca, doença de Crohn, tuberculose intestinal, insuficiência pancreática exócrina, gastrite atrófica, linfoma intestinal, anemia perniciosa, hipotireoidismo, gastrite autoimune e afecções neurológicas de causa não vascular.

Os resultados foram descritos em formulário de investigação apropriado.

A análise estatística foi realizada no software SPSS IBM $22 \mathrm{USA}^{\circledR}$. Foram calculadas medidas de tendência central e dispersão para as variáveis e correlatos descritivos, teste ChiQuadrado ou teste exato de Fisher (conforme apropriado) para as correlações, teste binomial para a variável Redução de vitamina B12 e análise de associação para prevalência das seguintes variáveis: Redução da vitamina B12 e Aumento de homocisteina. Foi realizada análise univariada para todas as variáveis, a fim de encontrar fatores de risco para a deficiência de vitamina B12. As variáveis com $\mathrm{p} \leq 0,05$ foram consideradas estatisticamente significativas e foram incluídas no modelo multivariado de regressão logística.

\section{RESULTADOS}

O número total de pacientes incluídos no estudo foi 116 . Desses, 55 (47,4\%) eram do sexo masculino e $61(52,6 \%)$ do sexo feminino. A média de idades do grupo feminino foi de 49,08 (DP: 15,631) e do masculino foi de 51,05 (DP: 16,598). Quanto à etnia, $10(8,6 \%)$ declararam-se brancos, $23(19,8 \%)$ negros e $83(71,6 \%)$ pardos.

Foi verificada uma taxa de redução de vitamina B12 em 10 pacientes $(8,6 \%)$ e um aumento de homocisteína em 11 (9,5\%). Em apenas 01 paciente verificou-se tanto redução de vitamina B12 quanto hiper-homocisteinemia.

Do grupo de pacientes com redução de vitamina B12 $(\mathrm{n}=10)$, $05(50 \%)$ eram do sexo masculino e $05(50 \%)$ eram do sexo feminino. A média de idades era de 53,2 para o grupo do sexo masculino (DP: 25,332) e 51,2 para o grupo do sexo feminino (DP: 10,232). Já no grupo de pacientes com aumento de homocisteína $(\mathrm{n}=11), 07(63,6 \%)$ eram do sexo masculino e $04(36,4 \%)$ eram do sexo feminino. A média de idade desses era de 51,14 para o grupo do sexo masculino (DP: 20,391) e de 56,5 para o grupo do sexo feminino (DP: 16,010). Dos 10 
pacientes em que foi detectada redução de vitamina B12, 4 (40\%) tinham idade maior ou igual a 60 anos. Por outro lado, dos 11 pacientes detectados com hiper-homocisteinemia, apenas 03 (27,3\%) incluíram-se nessa faixa etária. (Tabela 1)

Tabela 1. Relação entre etnia, sexo e idade com a redução do nível sérico de vitamina B12 e aumento do nível sérico de homocisteína.

\begin{tabular}{|c|c|c|c|c|c|c|c|c|}
\hline & & \multicolumn{2}{|c|}{ BRANCO } & \multicolumn{2}{|c|}{ PARDO } & \multicolumn{2}{|c|}{ NEGRO } & \multirow{2}{*}{$\begin{array}{l}\text { IDADE } \\
\text { MÉDIA }\end{array}$} \\
\hline & & Masculino & Feminino & Masculino & Feminino & Masculino & Feminino & \\
\hline \multirow{3}{*}{$\begin{array}{l}\text { REDUÇÃO DA VIT. } \\
\text { B12 }\end{array}$} & Sim & 1 & 0 & 3 & 3 & 1 & 2 & 52 \\
\hline & Não & 3 & 6 & 35 & 42 & 12 & 8 & 50 \\
\hline & Sim & 0 & 1 & 6 & 3 & 1 & 0 & 53 \\
\hline \multirow{2}{*}{$\begin{array}{l}\text { AUMENTO DA } \\
\text { HOMOCISTEINA }\end{array}$} & Não & 3 & 3 & 16 & 25 & 8 & 4 & 50 \\
\hline & FR & 1 & 2 & 16 & 17 & 4 & 6 & 49 \\
\hline
\end{tabular}

*FR: falta de reagente ${ }^{\mathrm{a}}$

a.dosagens comprometidas devido à falta do reagente no tempo do estudo

Considerando-se a variável etnia, o grupo de pacientes com redução de vitamina B12 obteve a seguinte distribuição: 01 branco (10\%), 06 pardos (60\%) e 03 negros (30\%). Da mesma forma, os valores encontrados no grupo de pacientes com hiper-homocisteinemia foi de 01 branco (09\%), 09 pardos $(82 \%)$ e 01 negro $(09 \%)$.

Dentre os fatores associados avaliados, foi visto que, dos pacientes com redução de vitamina B12 (n=10), 40\% apresentou afecção neurológica não explicada por causas vasculares, 20\% anemia macrocítica, 20\% alcoolismo, 20\% uso de inibidor de bomba de prótons, $10 \%$ uso de metformina, $10 \%$ dieta estritamente vegetariana, $10 \%$ diagnóstico de infecção por HIV, 10\% diagnóstico prévio de gastrite atrófica e 10\% diagnóstico prévio de hipotireoidismo.

De forma semelhante, dentre os pacientes que apresentaram aumento de homocisteína ( $\mathrm{n}=11$ ), 9,09\% apresentou afecção neurológica não-vascular, 45,4\% alcoolismo, 27,3\% uso de metformina, $18,1 \%$ uso de inibidor de bomba de prótons, 9,09\% diagnóstico de infecção por HIV e 9,09\% diagnóstico prévio de hipotireoidismo.

\section{DISCUSSÃO}

Sabe-se que a prevalência da deficiência de vitamina B12 pode variar de acordo com o parâmetro utilizado. Existem diversas definições de deficiência de B12, dependendo principalmente da população estudada e do kit disponível. ${ }^{13}$ Os testes de sensibilidade e especificidade ainda não foram capazes de definir um padrão ouro para definição de deficiência de vitamina B12. No futuro, novos kits de ensaio como o da holotranscobalamina devem substituir os kits atualmente usados. No presente momento, a deficiência de cobalamina é mais comumente definida como valores de B12 sérico < $200 \mathrm{pg} / \mathrm{ml}$ e/ou $150 \mathrm{pmol} / \mathrm{L}$, homocisteína $>13$ umol/L e/ou ácido metilmalônico $>0,4$ umol/L. ${ }^{13}$ No nosso estudo usamos os kits disponibilizados no Hospital Geral de Fortaleza, que tinham como valores de referência para B12 $<200$ pg/ml e para homocisteína $>0,203 \mathrm{mg} / \mathrm{dL}$.

Evidenciou-se na população geral de países industrializados uma prevalência média de deficiência de cobalamina de $20 \%$, variando de $5 \%$ a $60 \%$, dependendo dos critérios de definição de deficiência de cobalamina usados. ${ }^{6} \mathrm{O}$ estudo de Framinghan evidenciou prevalência de $12 \%$ entre os idosos que habitam a cidade. Usando a definição mais restritiva citada acima, semelhante à definição do nosso estudo, Andrès et al. evidenciou uma prevalência de 5\% em um grupo de pacientes internados em um hospital terciário. ${ }^{13}$ Em um outro estudo com desenho semelhante ao nosso, realizado em pacientes hospitalizados no Canadá, foi evidenciado uma prevalência de $3 \%$ de deficiência de cobalamina $(<138 \mathrm{pmol} / \mathrm{L})$ e de $13 \%$ de "deficiência intermediária" (138-221 pmol/L) ${ }^{13,14}$ No nosso estudo, a prevalência de deficiência de vitamina B12 ou de aumento de homocisteína foi de $18 \%$. Se analisados separadamente, a deficiência de cobalamina teve prevalência de $8,6 \%$, já a prevalência do aumento isolado de homocisteína foi de 9,5\%. Quando comparamos com a literatura mundial, a prevalência encontrada na nossa enfermaria se assemelha à da população geral mundial e é um pouco maior do que a prevalência encontrada nos pacientes internados em hospitais terciários.

A prevalência do nosso estudo foi semelhante em ambos os sexos, o que se assemelha aos dados encontrados em um estudo transversal mexicano com 288 famílias o qual demonstrou prevalência semelhante em ambos os gêneros. ${ }^{4}$

Nos EUA, Shipton et al. demonstraram a variação de deficiência de vitamina B12 de acordo com a idade, afetando cerca de $3 \%$ da população entre 20 e 39 anos, 4\% daqueles com idade entre 40 e 59 anos e $6 \%$ daqueles com mais de 60 anos. Quando se considerava a deficiência intermediária, entre $148 \mathrm{pmol} / \mathrm{L}$ e $221 \mathrm{pmol} / \mathrm{l}$, a prevalência foi de $15 \%$ naqueles com idade de 20-59 anos e de 20\% naqueles com idade superior a 60 anos. ${ }^{15}$ Outro estudo, com foco em idosos, principalmente os institucionalizados, doentes ou mal nutridos na França, evidenciou uma prevalência de 30 a $40 \%{ }^{13,16} \mathrm{Na}$ presente amostra, a prevalência de deficiência de cobalamina nos idosos, demonstrada pela redução dos níveis séricos de B12 ou pelo aumento da homocisteína, foi de $18 \%$.

Em relação à etnia, existem poucos estudos publicados que 
incluam a cor parda, a qual representa $60 \%$ da nossa casuística. $\mathrm{O}$ estudo que mais se aprofundou com investigação de etnia foi realizado nos EUA, e avaliou negros, brancos e descendentes hispânicos. Nessa pesquisa, os hispânicos representavam o grupo com menores níveis séricos de vitamina B12. ${ }^{16}$

No nosso estudo, de caráter transversal, a continuação da investigação etiológica não era um dos objetivos, sendo avaliados os fatores possivelmente associados no momento do diagnóstico de deficiência de cobalamina. Assim sendo, uma parte dos fatores associados permaneceram sem definição diagnóstica, mas corroboraram com a descrição do quadro clínico.

$\mathrm{Na}$ nossa casuística, 40\% dos pacientes com deficiência de vitamina B12 apresentavam afecções neurológicas não vasculares e $20 \%$ apresentavam anemia macrocítica. Sabe-se que os principais achados da deficiência de cobalamina são anemia megaloblástica e doença neurológica desmielinizante reversíveis, o que é semelhante aos nossos achados. ${ }^{17}$ Não havia nenhum paciente que apresentasse os dois achados concomitantemente. Esse dado foi condizente com o existente na literatura, que não evidencia associação de sintomas neurológicos com alterações hematológicas clínicas ou laboratoriais. ${ }^{15}$

Um estudo realizado na França com 172 idosos evidenciou em $70 \%$ dos pacientes algum sintoma neuropsiquiátrico e em 76\% alguma anormalidade hematológica. ${ }^{15}$ São valores maiores que os encontrados no nosso estudo, porém nossa casuística inclui todas as faixas etárias do adulto.

Esse mesmo estudo realizado na França, de desenho semelhante ao nosso, discriminou as principais etiologias identificadas nos idosos. A má-absorção se destacou em 53\%, a anemia perniciosa estava presente em $33 \%$, a baixa ingesta em $2 \%$ e apenas $1 \%$ foi pós-cirúrgica. ${ }^{13}$ Quando a população geral é estudada, a anemia perniciosa continua sendo a principal causa definida de deficiência de vitamina B12. ${ }^{17}$

O nosso estudo não prosseguiu a investigação com dosagem de anticorpos anti-fator intrínseco e anti-células parietais ou com endoscopia. É possível que muitos desses pacientes que apresentaram associação com afecções neurológicas não vasculares e anemia macrocítica fossem portadores de anemia perniciosa.

Assim sendo, na nossa casuística a má-absorção de vitamina B12 está como principal fator causal, já que $20 \%$ dos pacientes apresentavam como fator de risco alcoolismo, 20\% uso de inibidor de bomba de prótons, $10 \%$ uso de metformina e 10\% história de gastrite atrófica.

O álcool, cuja fisiopatologia na deficiência de vitamina B12 ainda não está bem clara, podendo ser por má nutrição ou piora na absorção intestinal, já foi descrito como fator de risco bem estabelecido, como no estudo polonês que estudou 71 homens alcoólatras e em todos eles encontrou alguma alteração, seja deficiência de folato, seja deficiência de vitamina B12, seja hiperhomocisteinemia. O mais comum era o aumento da homocisteína. ${ }^{18}$ Esse dado é compatível com nossos achados, já que dos pacientes com deficiência de vitamina B12, 20\% tinham o álcool como fator de risco associado. Já nos pacientes que tinham aumento da homocisteína, essa associação chegou a $45 \%$.

Outra observação interessante refere-se às causas de deficiência de vitamina B12 por má absorção, em que o álcool não é citado como importante fator do risco nos trabalhos analisados. Essa importância é dada para os inibidores de bomba de prótons, para as biguanidas, para gastrite atrófica, para gastrectomia, ressecção ileal, doença de Crohn e supercrescimento bacteriano. ${ }^{19} \mathrm{Na}$ nossa amostra, o alcoolismo aparece como um dos principais fatores de risco.

O uso da metformina como fator de risco bem definido para deficiência de B12 é bem estabelecido na literatura, como sugere uma coorte recente que identificou o risco de $11,2 \%$ maior em pacientes que usam metformina em dose superior a $500 \mathrm{mg} / \mathrm{dia}$, sugerindo inclusive a dosagem rotineira dessa vitamina em pacientes que fazem uso a longo prazo da biguanida. ${ }^{19}$ No nosso estudo, o uso desse fármaco correspondeu a $10 \%$ dos casos de deficiência de cobalamina e $27,3 \%$ quando avaliado o aumento da homocisteína. ${ }^{17}$

$\mathrm{O}$ uso de inibidores da bomba de prótons tem odds ratio de 1,83 para deficiência de vitamina B12, no seu uso por mais de 8 semanas, como demonstrado em recente metanálise. ${ }^{20}$ Entretanto, assim como a metformina, faltam dados que marquem sua real importância entre as causas de deficiência de vitamina B12. Na nossa casuística, se apresentou com uma prevalência em $20 \%$ dos casos.

A infecção pelo HIV já tem correlação estabelecida com a deficiência de B12. Um estudo realizado em um hospital terciário no sudeste do Brasil evidenciou que $6 \%$ dos pacientes infectados com esse vírus apresentavam deficiência de vitamina B12. ${ }^{21}$ Entretanto esse dado é conflitante, pois há evidências que essa deficiência pode ser encontrada em até $39 \%$ desses pacientes. ${ }^{13}$ De toda forma, o achado em $10 \%$ dos pacientes com redução dos níveis séricos de B12 é superior ao descrito na literatura.

A deficiência de cobalamina relacionada à dieta vegetariana na nossa casuística foi semelhante à encontrada na literatura. Assim como no hipotireoidismo, que foi visto em $10 \%$ dos nossos pacientes. Quando a pesquisa dos níveis séricos de cobalamina é feita no paciente sabidamente portador de hipotireoidismo, a deficiência de B12 pode ser encontrada em até $40 \%$ desses pacientes, sendo indicada a dosagem rotineira nesses pacientes. ${ }^{22}$

As correlações entre os fatores de risco investigados e a deficiência de vitamina B12 ou aumento de homocisteína apresentaram $p>0,05$, o que faz com que não sejam uma associação estatisticamente significativa.

A falta da dosagem da homocisteína em 46 pacientes e a não coleta de ácido metilmalônico em todos os pacientes foi um aspecto negativo do nosso trabalho, já que sabidamente eles 
aumentam a sensibilidade para o diagnóstico adequado de deficiência de cobalamina. ${ }^{13}$

Outra crítica que fazemos ao nosso estudo é a não realização de endoscopia, coleta de anticorpos anti-células parietais e anti-fator intrínseco para documentar a real prevalência de anemia perniciosa, principal causa de deficiência de vitamina B12.

A descrição da prevalência semelhante à população geral, porém maior que os dos pacientes internados ao redor do mundo nos levam a considerar que nossos pacientes são diagnosticados mais tardiamente, com manifestações neurológicas ou hematológicas mais graves. Provavelmente estamos falhando em detectar os pacientes com risco na atenção primária.

Esse estudo também levanta a possibilidade de existir em nosso meio uma importância maior do alcoolismo, do uso de biguanidas, de inibidor de bomba de prótons e do hipotireoidismo como causas de deficiência de vitamina B12 em nosso meio, o que pode sugerir uma propedêutica diagnóstica diferenciada para os nossos pacientes.

Diante dos dados verificados nesse estudo, sugerimos que os pacientes alcoólatras, usuários crônicos de inibidores de

\section{REFERÊNCIAS}

1. Herrmann W, Obeid R, Schorr H, Geisel J. Functional vitamin B12 deficiency and determination of holotranscobalamin in populations at risk. Clin Chem Lab Med. 2003;41(11):1478-88.

2. Herrmann W, Schorr H, Obeid R, Geisel J. Vitamin B-12 status, particularly holotranscobalamin II and methylmalonic acid concentrations, and hyperhomocysteinemia in vegetarians. Am J Clin Nutr. 2003;78(1):131-6.

3. Herrmann W, Geisel J. Vegetarian lifestyle and monitoring of vitamin B-12 status. Clin Chim Acta. 2002;326(1-2):47-59.

4. Allen LH, Rosado JL, Casterline JE, Martinez H, Lopez P, Muñoz E, et al. Vitamin B-12 deficiency and malabsorption are highly prevalent in rural Mexican communities. Am J Clin Nutr. 1995;62(5):1013-9.

5. Diez-Ewald M, Torres-Guerra E, Layrisse M, Leets I, Vizcaíno G, Arteaga-Vizcaíno M. Prevalence of anemia, iron, folic acid and vitamin B12 deficiency in two Bari Indian communities from western Venezuela. Invest Clin. 1997;38(4):191-201.

6. Carmel R. Current concepts in cobalamin deficiency. Annu Rev Med. 2000;51:357-75.

7. Carmel R, Green R, Rosenblatt DS, Watkins D. Update on cobalamin, folate, and homocysteine. Hematology Am Soc Hematol Educ Program. 2003:62-81.

8. Gutierrez M, Franques J, Faivre A, Koric L, Chiche L, Attarian $\mathrm{S}$, et al. [Diagnosis of vitamin B12 deficiency: a case illustrating diagnostic pitfalls]. Rev Neurol (Paris). French.

9. Carmel R. Measuring and interpreting holo-transcobalamin (holotranscobalamin II). Clin Chem. 2002;48(3):407-9. bomba de prótons ou de metformina, ou ainda portadores de hipotireoidismo sejam rastreados na atenção básica de saúde para deficiência de vitamina B12. Naqueles em que o rastreio for positivo, mas forem oligossintomáticos, medidas simples como abstinência alcoólica, substituição de medicamentos e ajuste de hormônio tireoidiano, associada à administração de dose de ataque de cobalamina, seguida de acompanhamento da recuperação e manutenção de níveis adequados de vitamina B12 são medidas fundamentais para evitar que complicações mais graves aconteçam futuramente. Caso a despeito das medidas houver queda dos níveis séricos de vitamina B12, recomenda-se iniciar investigação diagnóstica mais onerosa, incluindo endoscopia e dosagem de anticorpos.

Tais medidas seriam capazes de reduzir exames desnecessários a longo prazo, além de reduzir a quantidade de internação hospitalar de pacientes com graves manifestações de deficiência de vitamina B12.

Sugerimos, ainda, que sejam feitos mais estudos com maior número de participantes e grupo controle a fim de avaliar os fatores de risco associados, sugerindo uma propedêutica que permita que o paciente seja diagnosticado mais precocemente e reduza custos desnecessários para o Sistema Único de Saúde.

10. Holleland G, Schneede J, Ueland PM, Lund PK, Refsum H, Sandberg S. Cobalamin deficiency in general practice: assessment of the diagnostic utility and cost-benefit analysis of methylmalonic acid determination in relation to current diagnostic strategies. Clin Chem. 1999;45(2):189-98.

11. Sullivan LW, Herbert V. Studies on the minimum daily requirement for vitamin b12. Hematopoietic responses to 0.1 microgm. Of cyanocobalamin or coenzyme b12, and comparison of their relative potency. N Engl J Med. 1965;272:340-6.

12. Green R, Kinsella LJ. Current concepts in the diagnosis of cobalamin deficiency. Neurology. 1995;45(8):1435-40.

13. Dali-Youcef N, Andrès E. An update on cobalamin deficiency in adults. Q J Med. 2009;102(1):17-28.

14. Gudgeon P. Cavalcanti R. Folate testing in hospital inpatients. Am J Med. 2015;128(1):56-9.

15. Shipton MJ, Thachil J. Vitamin B12 deficiency - A 21st century perspective. Clin Med (Lond). 2015;15(2):145-50.

16. Hinds HE, Johnson AA, Webb MC, Graham AP. Iron, folate, and vitamin B12 status in the elderly by gender and ethnicity. J Natl Med Assoc. 2011;103(9-10):870-7.

17. Stabler SP. Clinical practice. Vitamin B12 deficiency. N Engl J Med. 2013;368(2):149-60.

18. Kopczyńska E, Ziółkowski M, Jendryczka-Maćkiewicz E, Odrowaz-Sypniewska G, Opozda K, Tyrakowski T. [The concentrations of homocysteine, folic acid and vitamin B12 in alcohol dependent male patients.] Psychiatr Pol. 2004;38(5):947-56. Polish. 
19. Oh R, Brown DL.Vitamin B12 deficiency. Am Fam Physician. 2003;67(5):979-86.

20. Jung SB, Nagaraja V, Kapur A, Eslick GD. Association between vitamin B12 deficiency and long-term use of acid-lowering agents: a systematic review and meta-analysis. Intern Med J. 2015;45(4):40916.
21. Castro L, Goldani LZ. Iron, folate and vitamin B12 parameters in HIV-1 infected patients with anaemia in southern Brazil. Trop Doct. 2009;39(2):83-5.

22. Jabbar A, Yawar A, Waseem S, Islam N, Ul Haque N, Zuberi L, et al. Vitamin B12 deficiency common in primary hypothyroidism. J Pak Med Assoc. 2008;58(5):258-61.

\section{Como citar:}

Cassimiro JM, Barreto CA Junior, Aguiar GP, Oliveira OJ. Deficiência de vitamina B12 em pacientes de uma enfermaria de clínica médica em Fortaleza/CE. Rev Med UFC. 2016 jan-jun;56(1):18-23. 